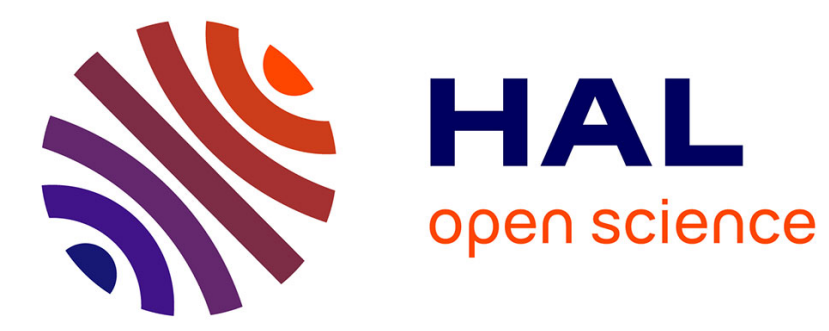

\title{
Storage-Enabled Access Points for Improved Mobile Performance: An Evaluation Study
}

Efthymios Koutsogiannis, Lefteris Mamatas, Ioannis Psaras

\section{To cite this version:}

Efthymios Koutsogiannis, Lefteris Mamatas, Ioannis Psaras. Storage-Enabled Access Points for Improved Mobile Performance: An Evaluation Study. 9th Wired/Wireless Internet Communications (WWIC), Jun 2011, Vilanova i la Geltrú, Spain. pp.116-127, 10.1007/978-3-642-21560-5_10 . hal01583635

\section{HAL Id: hal-01583635 \\ https://hal.inria.fr/hal-01583635}

Submitted on 7 Sep 2017

HAL is a multi-disciplinary open access archive for the deposit and dissemination of scientific research documents, whether they are published or not. The documents may come from teaching and research institutions in France or abroad, or from public or private research centers.
L'archive ouverte pluridisciplinaire HAL, est destinée au dépôt et à la diffusion de documents scientifiques de niveau recherche, publiés ou non, émanant des établissements d'enseignement et de recherche français ou étrangers, des laboratoires publics ou privés. 


\title{
Storage-Enabled Access Points for Improved Mobile Performance: An Evaluation Study *
}

\author{
Efthymios Koutsogiannis ${ }^{1}$, Lefteris Mamatas ${ }^{1}$, and Ioannis Psaras ${ }^{2}$ \\ 1 Democritus University of Thrace, \\ Dept. of Electrical and Computer Engineering/Space Internetworking Center, \\ 12 Vas. Sofias Str., 67100 Xanthi, Greece \\ 2 University College London, \\ Dept. of Electronic and Electrical Engineering, \\ WC1E 7JE, Torrington Place, London, UK \\ i.psaras@ee.ucl.ac.uk
}

\begin{abstract}
Due to existing networking paradigms and people communication habits, Internet is characterized by: (i) information that associates more and more with network edges, while corresponding traffic doesn't, (ii) a considerable amount of unexplored network resources that resides near mobile users, while their connectivity is usually poor and expensive in terms of financial cost and resource consumption.

To address the above challenges, we introduce a cooperative networking scheme where Home Access Points are equipped with storage capabilities and offer connectivity to mobile users. Whenever connectivity sharing is unavailable, the access points store the pending data and transmit it once connectivity becomes available. We explore how storage-enabled opportunistic routing algorithms can handle periods of intermittent connectivity. We experiment with realistic scenarios based on real network configurations and mobility maps. Our results demonstrate the potential of the proposed paradigm as well as guide us to define its follow-up evolution steps.
\end{abstract}

Keywords: user-centric networking, delay-tolerant networking, opportunistic routing

\section{Introduction}

Research on Opportunistic Mobile Networks and DTNs has been mainly concerned with storage and energy constraints for mobile devices and optimization of connectivity opportunities between encounters. The more the encounters and data-exchanges between the mobile nodes, the more the energy and

\footnotetext{
* The research leading to these results has received funding from the European Community's Seventh Framework Programme (FP7/2007-2013_FP7-REGPOT-2010-1, SP4 Capacities, Coordination and Support Actions) under grant agreement $n^{\circ}$ 264226 (project title: Space Internetworking Center-SPICE ).
} 
storage needed. The Delay-Tolerant and Opportunistic Networking research has focused mainly on mobile-only devices. That is, researchers have been investigating ways to optimize communication opportunities between the mobile devices themselves ${ }^{3}$. This is indeed the case in rural areas, for instance, where connectivity points to the network are rare, or in case of applications that do not require Internet connectivity (e.g., distributed online social networks [1]).

In this study, we focus on metropolitan environments, where there are plenty of connectivity points to the Internet. We extend the operational spectrum of Opportunistic Networks and DTNs and consider Home Access Points (APs or HAPs) as integral part of these networks. We consider storage a cheap resource that can easily be integrated in future APs and evaluate the performance of existing DTN protocols in this setting. In particular, given that connectivity opportunities to APs/HAPs and therefore the Internet now increase, we investigate whether and to which extend the storage and energy constraints can now be somewhat relaxed.

We motivate our study based on the following facts:

- New applications and people communication habits call for approaches that go beyond the traditional client-server model. Users are increasingly active in uploading content to social networking sites, for instance, or hosting data, e.g., in P2P file-sharing platforms. Moreover, it is shown that Internet traffic is growing faster at the network edge than at the core [2]. In other words, future networks call for user-centricity, where applications demand that content is closer to the user.

- Internet traffic forecasts show that mobile users are expected to double-up every year through $2014^{4}$. Similar studies show that the amount of usergenerated content is going to increase massively ${ }^{5}$ and furthermore, that most of it will be generated by mobile devices, e.g., smart-phones et simila. This urges the need for easy access to the Internet for mobile users, since content has to be readily available.

- Statistics show that the vast majority of households (e.g., $70 \%$ in UK) own a broadband connection ${ }^{6}$, which remains unexploited most of the day. It is obvious that an area of dense connectivity can be formed if the HAPs' network and storage resources are being shared between home- and mobileusers.

- User-Provided Networks and connectivity sharing schemes have already been investigated and implemented (e.g., FON [3]). In our previous studies [4], [5] we have presented algorithms for connectivity sharing among home- and guest-users and proved that careful design can guarantee seamless connectivity sharing for the home-user.

\footnotetext{
${ }^{3}$ Vehicular DTNs [6] and Infostations [7] can be considered as exceptions here, since these deployments are not constrained by energy and/or storage depletion in most cases

${ }^{4}$ http://www.cisco.com/en/US/netsol/ns827/networking_solutions_sub_solution.html

${ }^{5}$ http://newsroom.cisco.com/dlls/2009/prod_102109.html

${ }^{6}$ http://www.statistics.gov.uk/cci/nugget.asp?ID=8
} 
In this study, we explore the performance benefits of extending the users' connectivity graph to include neighbor HAPs, exploiting this way local unused resources. An occupied HAP that temporarily cannot forward packets from guestusers to the Internet may store the packets until a connection opportunity is available. The stored packets can be forwarded to another HAP in range, or relayed to a mobile node that may find another connectivity point.

We focus on the evaluation of DTN routing protocols, when the opportunistic network infrastructure is extended to also include HAPs, whenever and wherever these are available. We have built a realistic scenario, where mobile users walk around the center of London. We assume that existing FON subscribers (i.e., we used the database from maps.btfon.com) employ the proposed paradigm. We apply different usage scenarios to the home-users (e.g., periodic use, away for the day, away for work) in order to emulate their on-off behavior in terms of connectivity offering. We assume that whenever the home-user is transmitting/receiving data the HAP is not available to the guest user. Therefore, the connection is available for guests whenever the home connection is idle. At this stage, we explore how existing opportunistic routing algorithms behave in this particular context; we evaluate the following well-known algorithms: Epidemic [8], First contact [9], Spray and Focus [10], Spray and Wait [11] and MaxProp [12]. We elaborate on these results in order to draw design guidelines for new algorithms, especially designed and optimized for the proposed paradigm. Our target is to identify the necessary properties that need to be integrated into opportunistic routing protocols in order to operate as efficiently as possible under this new setting, i.e., when storage resources are part of the HAP and are offered to mobile users. We leave these designs as our immediate future work task.

Our findings indicate that: (i) integrating storage capabilities to HAPs substantially increases connectivity opportunities for mobile nodes, (ii) service quality for mobile users increases with the number of available HAPs and their respective storage resources, (iii) there is a lot of space for improvement for opportunistic routing protocols, performance-wise, (iv) among the evaluated algorithms Spray and Focus is the most efficient, since it balances better between performance and overhead, and (v) an efficient routing algorithm for this new networking paradigm could offload routing sophistication or routing state info from mobile devices to HAPs, in order to reduce energy consumption at mobile users, without compromising performance.

In Section 2, we discuss the related work in terms of other broadband access sharing schemes and opportunistic routing algorithms. In Section 3, we describe our experimental setup and the associated methodology and in Section 4 we present our experimental results. We conclude the paper in Section 5, where we also discuss our future work.

\section{Related work}

Broadly speaking, our study balances between User-Provided Networks and Opportunistic or Delay-Tolerant-Networks. Below, we briefly provide background, 
state of the art information on these areas and highlight how the present study advances research beyond the state of the art.

\subsection{State of the Art}

User-Provided Networks. UPNs have been recently proposed [13] as an umbrella framework for Broadband Access Sharing. Citywide Ubiquitous Wi-Fi Access sharing issues have been investigated in the past [14] and have shown that commercial deployment of such initiatives is indeed possible.

Famous commercial deployments include the FON Community, the OpenSpark Community and the Wifi.com ${ }^{7}$. Each of these deployments has a different starting point, incentive-wise, and therefore, comprises different scalability and efficiency properties. For instance, Wifi.com is based on a social networking approach, while FON is based on a business model that explicitly allocates resources to home- and guest-users. Recent studies have also investigated the wireless interface's capabilities to share resources [15].

The success of the above initiatives clearly shows that users are keen on giving away a portion of their bandwidth in order to have access when out of home or office. In our opinion, whatever the Broadband Access Sharing scheme, it has to balance between two fragile service-points: (i) the guarantee to the homeuser for seamless sharing, performance-wise and (ii) the mobile-users' quality of experience. The former point has been our focus in previous studies [4], [5]. The latter constitutes our target in the present study, where we elaborate on the functionality of an efficient routing mechanism in order to achieve lower latency and reduced transmission redundancy. Hence, our target is increased responsiveness, efficient storage management and longer battery life for mobile devices.

Opportunistic Networks and DTNs. A lot of research has taken place recently to deal with routing issues in intermittently connected networks. Here, we briefly present the most prevalent DTN routing algorithms.

In Epidemic routing algorithm [8], a node copies a message to every other node it encounters. Variations of this algorithm include Randomized flooding and Utility-based flooding. Randomized flooding or Gossiping (random-flood) is quite similar to Epidemic although each message is copied with probability $p<1$. In the Utility-based flooding, a message is copied only if the node encountered has a utility value higher than the current by some threshold $U_{t h}$.

MaxProp [12] is another flooding-based mechanism which removes the messages once a copy is delivered. Moreover, MaxProp prioritizes both messages to be transmitted and dropped based on previous encounters.

Resource Allocation Protocol for Intentional DTN (RAPID) [16] treats DTN routing as a resource allocation problem. It uses a utility function that: (i) assigns a value based on the metric being optimized to every packet and (ii) first replicates packets that increase the utility function.

\footnotetext{
${ }^{7}$ URLs: http://www.btfon.com, http://open.spark.fi, http://www.wifi.com
} 
Other approaches use a controlled replication or spraying. In Spray and Wait [11], a small fixed number of copies are distributed (sprayed) to different relays (i.e., the first few encountered). Each relay waits until it encounters the destination itself. A more sophisticated variation is Spray and Focus [10] which operates like Spray and Wait during spraying. Each relay, instead of waiting, can forward its copy to a potentially more appropriate relay, using a utility-based scheme.

For a complete survey on DTN routing protocols, we refer the reader to [17] and [18]. In addition, related work on exploiting WLAN hotspots in order to enhance opportunistic web access and offload cellular traffic can be found in [19] and [20], respectively.

\subsection{Progress Beyond the State of the Art}

To release the boundaries that keep these two research fields separated and unleash the communication opportunities of the corresponding framework, we highlight the following:

- UPNs exploit connectivity sharing opportunities only. Broadband access is only one of the available resources that can be shared between home and mobile users. In this study, we explore the potential of sharing storage resources as well, given that storage is available at the HAPs. That is, we extend the sharing capabilities of UPNs to include storage resources as well.

- DTN routing research has been mainly concerned with encounters between mobile nodes only. Although in case of DTN nodes, both connectivity and storage resources are shared between the nodes, they do not consider collaborating with fixed points of the infrastructure. Here, we extend this trend to a boundless framework, where mobile nodes can also take advantage of both connectivity and storage resources of HAPs.

\section{Experimental Setup and Methodology}

We simulate a realistic mobility setup. We have chosen a geographical area in the center of London sized $2160 \mathrm{~m}$ x $1600 \mathrm{~m}$, see Fig. 1. This area covers 349 different streets and 1876 landmarks. We have gathered detailed information from [3] regarding the exact position and the number of BTFON users in this particular area (i.e., 118 users). We parsed all this information into the ONE simulator [21].

We extended the ONE simulator with better support for wired networks and two new types of nodes: (i) the home-user that owns a HAP, and (ii) a server node, which hosts data for the mobile users (e.g., social profile photos). We assume that all home-users are permanently connected to a server node (e.g., a server farm) situated in Gower Street (see Fig. 1).

Mobile nodes are moving in the area and transmit periodically data (every 60 to 80 seconds, according to a uniform distribution) to the server node. Message sizes follow the uniform distribution [100kB, 200kB] (e.g., a typical social profile 
photo). Their movement is restricted to the streets in the map, according to the map route movement model and the shortest path algorithm in ONE simulator.

All nodes (mobile and static) have storage capabilities, but static nodes, i.e., HAPs, comprise extensive storage resources. In our setup, mobile nodes and HAPs have $30 \mathrm{MB}$ of storage available. These are only indicative figures and serve as pointers for future settings. As already mentioned, the HAP is only available to mobile users, whenever the home-user is idle. This practically means that when the home-user is using his Internet connection, only storage capabilities are offered to the mobile nodes within range. We assume three representative home-user profiles: (i) users that are idle for 8 hours per day only (e.g., during working hours), (ii) users that are permanently idle (e.g., they are absent), and (iii) users that are only periodically using their connection throughout the day (i.e., according to a normal probability distribution: 1-3 hours at home and 12 hours away). Nodes periodically transmit beacons to recognize each other's presence. Fig. 1, shows our experimental setup. The server is referenced as snode, mobile-guests as g-nodes and home-users as h-nodes.

At this stage of investigation, we evaluate how existing opportunistic routing algorithms behave in this particular scenario, where HAPs offer storage resources apart from only connectivity. We selected five representative storage-enabled algorithms, namely: Epidemic [8], First contact [9], Spray and Wait [11], Spray and Focus [10] and MaxProp [12]. Our target is to identify key requirements and design guidelines for a customized algorithm that exploits efficiently not only connection opportunities, but storage resources as well.

Since a crucial aspect is to maximize energy efficiency of the mobile nodes, a maximum data delivery ratio should be combined with minimum communication / storage overhead and latency. In this context, we measure the following:

- OverheadRatio $=\frac{\text { PacketsRelayed-PacketsReceived }}{\text { PacketsReceived }}$, which captures packet delivery ratio with respect to the number of packets relayed.

- AverageLatency, which captures the average packet latency that increases with the number of hops the packet crosses.

- AverageBufferTime, which reflects the average time the packets are buffered, in order to evaluate protocol storage efficiency.

\section{Experimental Results}

The experimental scenarios presented in the next section were designed in order to allow for:

- Studying the impact of the proposed extended networking scheme on the performance of a representative opportunistic routing algorithm. In this context, the Epidemic ${ }^{8}$ protocol was evaluated in various deployment levels by increasing the number of HAPs in Scenario 1. In Scenario 2, evaluation is based on different usage profiles that home-users adopt.

\footnotetext{
${ }^{8}$ Epidemic protocol was selected for these scenarios and more specifically EpidemicOracle version, which upon message delivery removes that message from all nodes.
} 


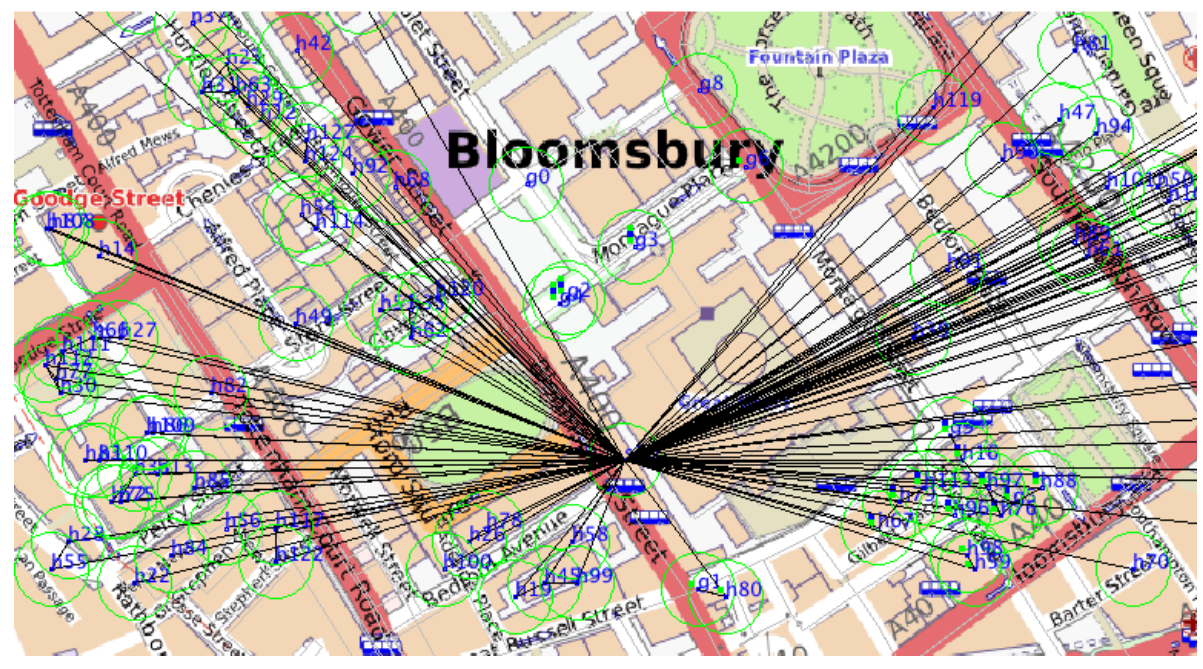

Fig. 1. Experimental Scenario

- Evaluating well-known DTN routing protocols' functionality used for communication between mobile nodes and static infrastructure. More specifically, we study their performance while scaling the proposed scheme with respect to mobile nodes' number, in Scenario 3. In scenario 4, we focus on the efficiency of the protocols with regards to guest-users' speed.

\subsection{Scenario 1: Impact of Home-Users' Number.}

The purpose is to show the advantages of the proposed framework, i.e., the extension of mobile network's boundaries to also include storage-equipped HAPs. In this first scenario, we explore the performance of a representative opportunistic routing protocol and more specifically Epidemic with 100 mobile-users, where the availability of HAPs is gradually increased (0-100). Home-users can be perceived as points of guaranteed message delivery to the destination server.

We observe in Fig. 2(a), that as the number of home-users increases, the average Latency declines, since less time is needed to reach a connectivity point to the Internet. Consequently, the average buffering time declines as well, as is shown in Fig. 2(b), owing to timely message delivery. The peak in measurements (i.e., Figures 2(a), 2(b)) for 5 home-users can be justified as follows: most of the first five HAPs in our database are situated in peripheral streets. Hence, they have low interaction probability with mobile nodes and consequently they do not get involved in the replication process. Therefore, average buffering time increases (Fig. 2(b)), since mobile nodes need to carry data for more time. Clearly, there are still more delivery probabilities than in the case of zero home-users (Fig. $2(\mathrm{a}))$, where the server is the only data delivery point. In conclusion, increasing the availability of storage-equipped HAPs enhances network's connectivity, thus average Latency and Buffering time decrease. 


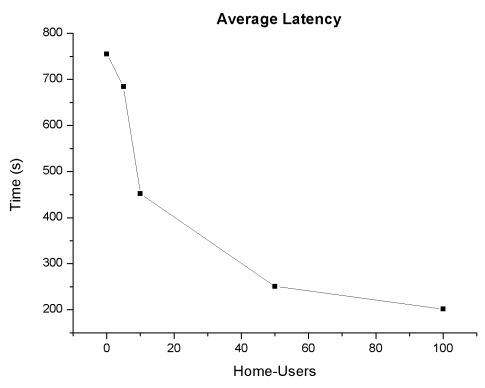

(a) Average Latency

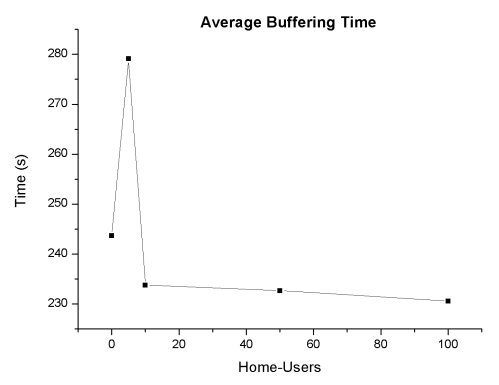

(b) Average Buffering Time

Fig. 2. Scenario 1: Impact of Home-Users' Number

\subsection{Scenario 2: Impact of Home-Users' Usage-Profiles.}

Here, each home-user adopts one of the three usage-profiles described in Section 3.1. This scenario intends to study the behavior of a representative opportunistic routing protocol in a topology consisting of 118 home-users with different usageprofiles. We discriminate the cases according to home-users' percentage that follows each of the three usage profiles: A) away for 8 hours, B) always away, C) periodically away (1-3 hours at home, then 1-2 hours away). Moreover, we simulated the cases where (A, B, C) percentages are: i) $(0,100,0)$, ii) $(20,40$, $40)$, iii) $(10,10,80)$, iv) $(0,0,100)$, while guest-users vary $(10-100)$.

We conducted a series of experiments where increasing home users with usage profiles (i) and (iii), causes lack of connectivity, while increasing the number of guest-users allows for more routes and improved message delivery in terms of time. In these experiments, we observed that data remains stored at homeusers available for: i) delivery to the server when connection will be up, ii) transmission to a guest-user in the vicinity, iii) transmission to a home-user in the vicinity. Fig. 3(b) presents average buffering time, which decreases as mobile nodes increase, while the four graphs coincide. Clearly, usage-profile has small impact on buffering time, since the number of home-users is sufficiently large. On the contrary, average Latency graphs vary, and their values increase when periodic nodes increase, while increasing guest-users decreases average Latency as depicted in Fig. 3(a). We report, although not present it here due to space limitations, that Overhead ratio is not influenced by usage-profile. Usage-profiles appear to have significant impact on average Latency, while Epidemic protocol manages to deliver messages, though with a high overhead. 


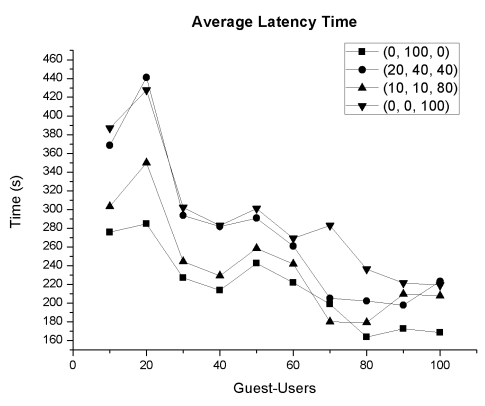

(a) Average Latency

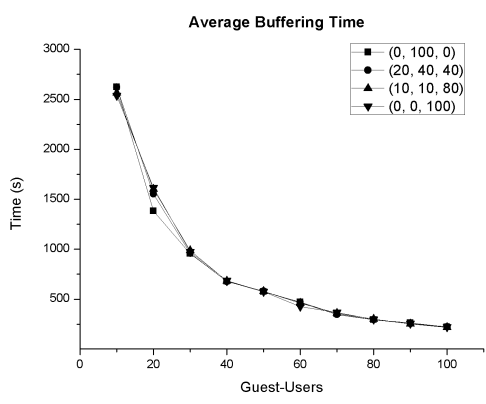

(b) Average Buffering Time

Fig. 3. Scenario 2: Impact of Home-Users' Usage-Profiles

\subsection{Scenario 3: Impact of Guest-Users' Number.}

In this scenario, we aim to compare the functionality of all five protocols and highlight the most efficient strategy, in the extended opportunistic networking paradigm introduced herein. The topology consists of 118 HAPs constantly available, while mobile nodes vary (10-100).

Spray and Wait introduces the highest average Buffering time (see Fig. 4(b)), which is not affected by the number of guest-users, while Spray and Focus achieves the lowest. Both algorithms were configured with 6 message copies, therefore their behavior at spraying phase is identical; consequently, the focus phase is responsible for the significant reduction of buffering time, as opposed to the wait phase. Fig. 4(a), depicts the average Latency, where Spray and Focus is again outperforming the rest of the protocols. First contact is the worst performing algorithm, since the first encountering node has no guarantee to deliver the message immediately. The Overhead ratio in Fig. 4(c) evinces the efficiency of Spray and Focus mechanism compared to the others. As expected Epidemic did not perform well with regards to overhead owing to mere flooding.

Therefore, increasing guest-users' number improves the average latency, except from First Contact. Furthermore, it also reduces average buffering time at the expense of overhead. In summary, regarding the impact of guest-users' number, Spray and Focus is the most efficient of the tested algorithms.

\subsection{Scenario 4: Impact of Guest-Users' Speed.}

The speed of mobile nodes, together with connectivity opportunities, the message size and links' bandwidth are critical to protocol's efficiency. Increased speed allows for more encounters with other nodes, although it reduces the communication time. Using realistic settings, we evaluate the performance of the five 


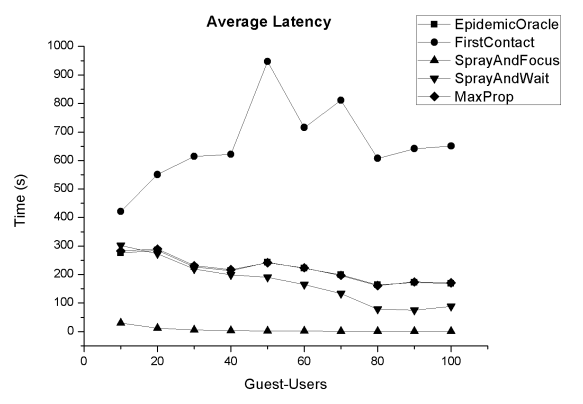

(a) Average Latency

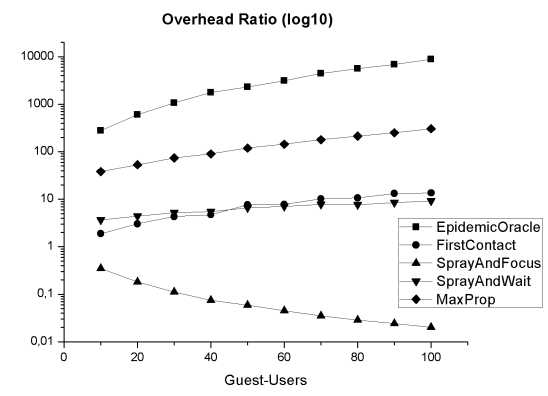

(c) Overhead Ratio

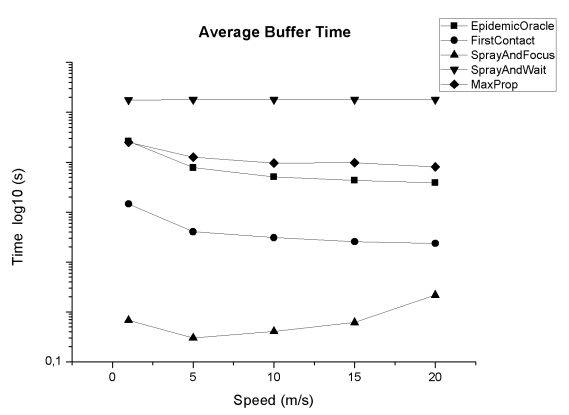

(e) Average Buffering Time

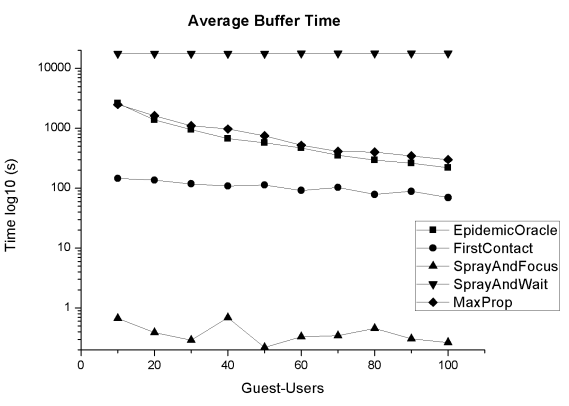

(b) Average Buffering Time

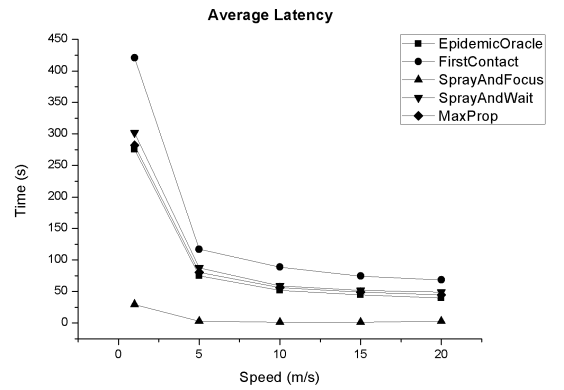

(d) Average Latency



(f) Overhead Ratio

Fig. 4. Scenario 3: (a), (b), (c), Scenario 4: (d), (e), (f) 
protocols with regards to speed differentiation, for 10 guest- (maximum supported number for the specific configuration) and 118 home-users available.

Increasing speed ( 1 to $20 \mathrm{~m} / \mathrm{s}$ ) allows for increased data relaying due to more encounters and reduces the average buffering time (Fig. 4(e)). Spray and Focus has the best performance, even though it is the only algorithm whose buffering time increases with speed; this is due to utility function's inability to adapt to increased encounters. This result shows the potential improvement of the specific algorithm in this context. Average latency in Fig. 4(d) is highly decreased for low speeds $(5 \mathrm{~m} / \mathrm{s})$, while further speed increase does not affect the results. Fig. 4(f) depicts the overhead ratio, where Epidemic routing has the highest overhead ratio in contrast to Spray and Focus. In conclusion, Spray and Focus is the best performing algorithm in this scenario with regards to all evaluation parameters.

\section{Conclusions}

We have proposed and investigated a cooperative networking scheme, in the context of User-Provided Networks, where storage resources are offered to mobile users, until bandwidth at HAPs becomes available to transmit mobile users' data. Storage-enabled nodes and access points are a key feature of our opportunistic networking paradigm. We evaluated the impact of this scheme on the performance of opportunistic routing and compared the performance of five wellknown DTN routing protocols in a realistic simulation setting. The proposed networking scheme avails in terms of latency and buffering time, although overhead ratio is sometimes increasing. Since mobile devices are involved, an efficient routing protocol that achieves high delivery ratio and low overhead is crucial. We have concluded that Spray and Focus [10] is the most suitable for the aforementioned scheme, amongst the evaluated protocols.

These preliminary results indicate that a new DTN routing protocol suitable for this environment can achieve improved overall performance. Its functionality should allow for reduced latency, communication and storage overhead, in conjunction with high delivery ratio, even in case of opportunistic routing (few and sparse HAPs). We argue that the basic guidelines for the design of the algorithm are: (i) Route data through HAPs with a higher probability than mobile nodes in order to alleviate the latter's storage and energy constraints. (ii) Exploiting HAP density and offloading sophistication or routing state info from mobile nodes to HAPs will further enhance performance e.g., via encounter prediction. (iii) Integration of Spray and Focus functionality in order to perform efficiently when mobile nodes are dominant in the topology. (iv) Dynamic configuration of message redundancy according to feedback from encounters and time scales [22], will allow for low overhead in conjunction with the desired delivery rate. As a next step we intend to integrate these observations in a new protocol.

\section{References}

1. Xu, T., Chen, Y., Fu, X., Hui, P.: Twittering by cuckoo: decentralized and socioaware online microblogging services. SIGCOMM Computer Communication Review 
40 (4), 473-474 (August 2010).

2. Leavitt, N.: Network-Usage Changes Push Internet Traffic to the Edge. IEEE Computer Magazine 43(10), 13-15 (Oct. 2010).

3. BT FON FON Wireless Ltd and British Telecommunications. http://www.btfon.com.

4. Psaras, I., Mamatas, L.: On Demand Connectivity Sharing: Queuing Management and Load Balancing for User-Provided Networks. Elsevier Computer Networks (COMNET), Special Issue on Wireless for the Future Internet, accepted (2010).

5. Mamatas, L., Psaras, I., Pavlou, G.: Incentives and Algorithms for Broadband Access Sharing. 1st ACM Sigcomm Workshop on Home Networks (HomeNets), New Delhi, India (August 2010).

6. Bychkovsky, V. et al.: A Measurement Study of Vehicular Internet Access Using in Situ Wi-Fi Networks. In Proceedings of MobiCom, 50-61, New York, USA, (2006).

7. Goodman, D.J., Borras, J., Mandayam, N.B., Yates, R.D.: INFOSTATIONS: a new system model for data and messaging services. In IEEE VTC'97, volume 2, pages 969-973 (May 1997).

8. Vahdat, A., Becker, D.: Epidemic routing for partially connected ad hoc networks. Technical Report CS-200006, Duke University (2000).

9. Fall, K.: A delay-tolerant network architecture for challenged internets. SIGCOMM '03, ACM, New York, NY, USA, 27-34. DOI=10.1145/863955.863960 http://doi.acm.org/10.1145/863955.863960

10. Spyropoulos, T., Psounis, K., Raghavendra, C.S.: Spray and Focus: Efficient Mobility-Assisted Routing for Heterogeneous and Correlated Mobility. Fifth Annual IEEE International Conference on Pervasive Computing and Communications (PerCom), 79-85, White Plains, NY (19-23 March 2007).

11. Spyropoulos, T., Psounis, K., Raghavendra, C. S.: Spray and wait: Efficient routing in intermittently connected mobile networks. In Proceedings of ACM SIGCOMM workshop on Delay Tolerant Networking (WDTN) (2005).

12. Burgess, J., Gallagher, B., Jensen, D., Levine, B. N.: MaxProp: Routing for VehicleBased Disruption-Tolerant Networks. In Proc. of IEEE INFOCOM (April 2006).

13. Sofia, R., Mendes, P.: User-provided Networks: Consumer as Provider. IEEE Communications Magazine 46(12), 86-91 (December 2008).

14. Thraves, C. et al.: Driving the Deployment of Citywide Ubiquitous WiFi Access. In SimulWorks (2008).

15. Solarski, M. et al.: An Experimental Evaluation of Urban Networking using IEEE 802.11 Technology. In Wireless Mesh, 1-10 (2006).

16. Balasubramanian, A., Levine, B.N., Venkataramani, A.: DTN routing as a resource allocation problem. In Proc. ACM SIGCOMM (August 2007).

17. Small, T., Haas, Z.: Resource and performance tradeoffs in delay-tolerant wireless networks. In Proceedings of ACM SIGCOMM WDTN (2005).

18. Psaras, I., Wood, L., Tafazolli, R.: Delay-/Disruption-Tolerant Networking: State of the Art and Future Challenges Technical Report (2009), available at: http://www.ee.ucl.ac.uk/ uceeips/dtn-srv-ipsaras.pdf

19. Pitkänen, M., Kärkkäinen, T., Ott, J.: Opportunistic Web Access via WLAN Hotspots. In Proc. of PERCOM '10, Mannheim, Germany, March 2010.

20. Han, B. et al.: Cellular traffic offloading through opportunistic communications: a case study. In Proceedings of ACM CHANTS, 2010.

21. Keränen, A., Ott, J., Kärkkäinen, T.: The ONE Simulator for DTN Protocol Evaluation. SIMUTools '09, Rome, Italy (2009).

22. Karvo, J., Ott, J.: Time scales and delay-tolerant routing protocols. In Proceedings of the 3rd ACM CHANTS '08, ACM, New York, NY, USA, 33-40 (2008). 